\title{
A fourth-order seven-point cubature on regular hexagons
}

\author{
Daniel Lee and Hui-Chun Tien
}

\begin{abstract}
We investigate the central moments of (regular) hexagons and derive accordingly a discrete approximation to definite integrals on hexagons. The seven-point cubature rule makes use of interior and neighbor center nodes, and is of fourth order by construction. The result is expected to be useful in two-dimensional (open-field) applications of integral equations or image processing.
\end{abstract}

\section{Introduction}

Hexagonal grids are of interest in some research studies [6, 8].

Hexagonal subregions are adopted in numerical simulation in the study of the origin of $\mathrm{U}$ waves in human heart electrophysiology (electrocardiograms (ECGs)) [6]. A slice of the left ventricular myocardium is mapped with a set of hexagonal grid (cells) and proper electrophysiological action potential (AP) is assigned to each cell. Since the timing of the APs follows a simulated excitation sequence, there are potential differences between cells' APs and dipole source vectors are generated. Then the ECG can be calculated as the sum of the weighted contributions of the dipole source vectors. However, this is a purely algebraic approach to obtaining ECG phenomena. With the inclusion of a reaction-diffusion system, numerical simulation of cardiac electrophysiology is conducted [8] in a reversed C-type domain which is approximated by hexagonal finite volumes. We consider in this work numerical cubature on regular hexagons and develop a fourth-order seven-point rule. The result can be applied to integral relations in approximated two-dimensional (irregular) domains, especially to problems of type of open field. Our main result, estimating the integral on a regular hexagon by using both interior and neighbor cell centers with the simple setup of having prescribed hexagonal nodes (or knots), seems previously unknown $[\mathbf{1}-\mathbf{5}, \mathbf{7}, \mathbf{9}]$.

As for the remaining sections, we introduce general configurations of (regular) hexagons and our main result in $\S 2$, resolve moments of a hexagon in $\S 3$, and determine the weights of a fourth-order cubature rule in $\S 4$. Conclusions are drawn in the final section. The Appendix includes some barycentric type trigonometric identities.

\section{The configurations and main result}

In two-dimensional applications of domains consisting of, or approximated by, (subsets of) Cartesian type regular hexagons, we denote the radius of a typical hexagon by $r$, the height by $h(=(\sqrt{3} / 2) r)$, and the center-to-center distance by $d(=2 h)$. The area of a hexagon is $|\Omega|=(3 \sqrt{3} / 2) r^{2}=2 \sqrt{3} h^{2}$. At a hexagon $\Omega$ in general configuration, we consider the center,

Received 22 January 2015; revised 23 July 2015.

2010 Mathematics Subject Classification 65D32, 35J05, 15A06 (primary). 


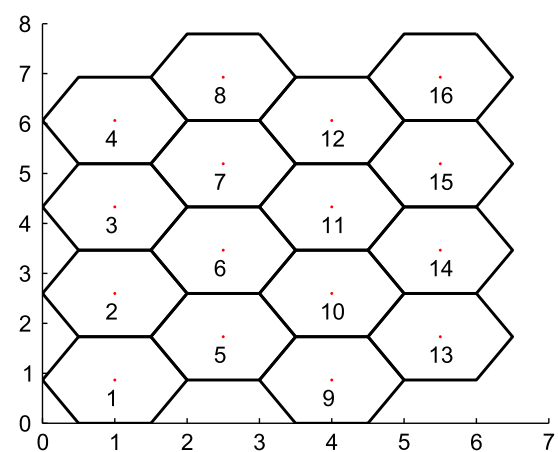

(a) Type I lattice.

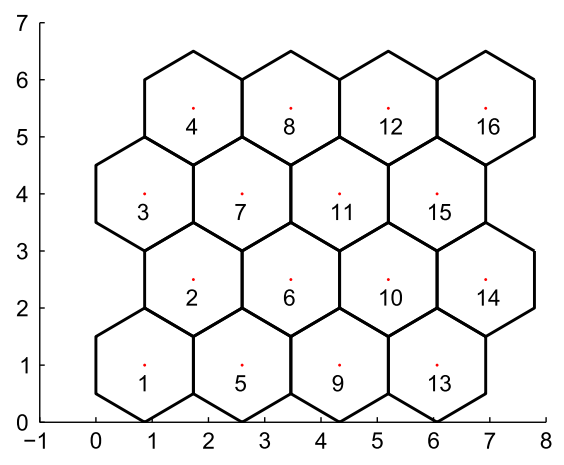

(b) Type II lattice.

FiguRE 2.1. Lattice of hexagons in natural order by columns.

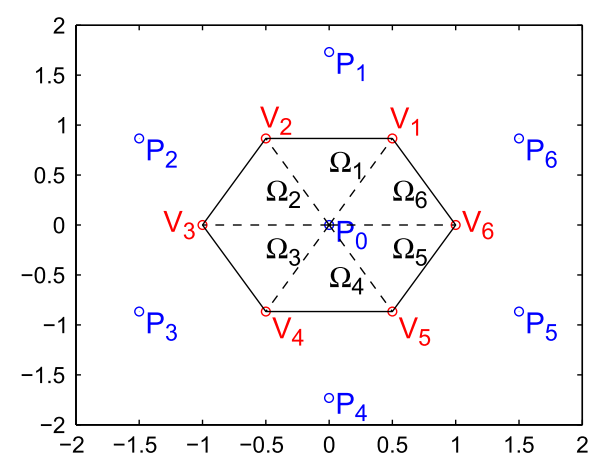

(a) Type I neighborhood.

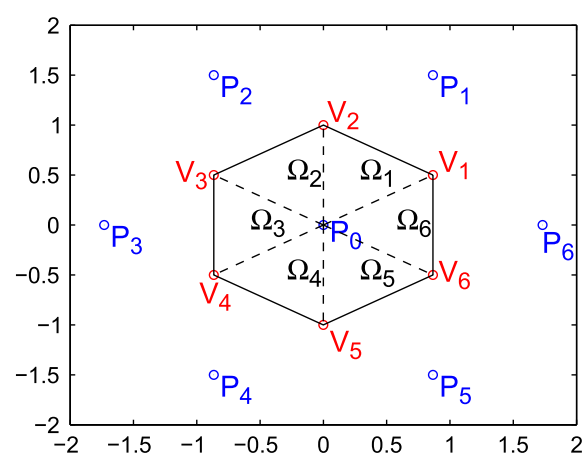

(b) Type II neighborhood.

FiguRE 2.2. Hexagonal finite-volume neighborhood.

$P_{0}=\left(x_{0}, y_{0}\right)$, and the vertices and neighbor (center) nodes,

$$
\begin{gathered}
V_{k}=\left(x_{0}, y_{0}\right)+r\left(\cos \theta_{k}, \sin \theta_{k}\right), \quad \theta_{k}=\varphi+\frac{k \pi}{3}, \quad 1 \leqslant k \leqslant 6, \\
\left(x_{k}, y_{k}\right)=P_{k}=\left(x_{0}, y_{0}\right)+d\left(\cos \xi_{k}, \sin \xi_{k}\right), \quad \xi_{k}=\psi+\frac{k \pi}{3}, \quad 1 \leqslant k \leqslant 6 .
\end{gathered}
$$

We assume $V_{7}=V_{1}$ and $P_{7}=P_{1}$ for convenience. Here the phase $\varphi$ is the start angle of local vertices, while $\psi=\varphi+\pi / 6$ is the start angle of neighbor (center) nodes. We regard $\varphi$ as the configuration parameter. Two particular instances are called type I $(\varphi=0)$ and type II $(\varphi=-\pi / 6)$ for convenience. Hexagon centers in lattices of these types are indexed as for an orthogonal Cartesian mesh as shown in Table 2.1. Indexing rules are visualized in Figures 2.1 and 2.2 .

\begin{tabular}{|c|c|c|}
\hline Phase angle & Type I, $\varphi=0$ & Type II, $\varphi=-\pi / 6$ \\
\hline Center point & $i^{\text {even }}$ & $j^{\text {even }}$ \\
\hline$c x(i, j)$ & $(1.5 i-0.5) r$ & $(2 i-1) h$ \\
\hline$c y(i, j)$ & $(2 j-1) h$ & $(1.5 j-0.5) r$ \\
\hline
\end{tabular}

TABLE 2.1. Lattices of type I and II hexagons. 
Only regular hexagons are considered in this work. A hexagon is at standard position if it is centered at the origin, that is, $\left(x_{0}, y_{0}\right)=(0,0)$. The following property is obvious.

Property 2.1 (Domain symmetry). Assumed at standard position,

(i) a hexagon is invariant under the reflection with respect to its center;

(ii) a type I or II hexagon is symmetric with respect to both the $x$ - and $y$-axes.

For a hexagon in general phase, we consider the partition (Figure 2.2)

$$
\Omega=\bigcup_{1 \leqslant l \leqslant 6} \Omega_{l} \equiv \bigcup_{1 \leqslant l \leqslant 6} \triangle P_{0} V_{l} V_{l+1}
$$

in which a typical component $\Omega_{l}$ is represented as a positively oriented (closed) triangle $P_{0} V_{l} V_{l+1}$. The relations

$$
\begin{aligned}
& \cos \left(2 \xi-\theta_{j}\right)=\cos \left(2 \xi-\varphi-\frac{j \pi}{3}\right)=\cos \left((2 \xi-\varphi)+\frac{(6-j) \pi}{3}\right) \\
& \sin \left(2 \xi-\theta_{j}\right)=\sin \left(2 \xi-\varphi-\frac{j \pi}{3}\right)=\sin \left((2 \xi-\varphi)+\frac{(6-j) \pi}{3}\right)
\end{aligned}
$$

translate into the following property.

PROPERTY 2.2 (Reflectional symmetry between two hexagons). The reflection with respect to the line $y=x \tan \xi$ in the $x y$-plane results in interchanging two hexagons at standard position and the associated two ordered lists,

$$
\left\{\varphi, V_{j}, P_{j}, \Omega_{j}, j=1,2, \ldots, 6\right\} \quad \text { and } \quad\left\{(2 \xi-\varphi), V_{\sigma(j)}, P_{\tau(j)}, \Omega_{\tau(j)}, j=1,2, \ldots, 6\right\} .
$$

Here the order-two permutations $\sigma(j):=6-j \% 6$ and $\tau(j):=1+((5-j \% 6)-1) \% 6$ are, in detail,

$$
\sigma=\left(\begin{array}{llllll}
1 & 2 & 3 & 4 & 5 & 6 \\
5 & 4 & 3 & 2 & 1 & 6
\end{array}\right) \text { and } \tau=\left(\begin{array}{llllll}
1 & 2 & 3 & 4 & 5 & 6 \\
4 & 3 & 2 & 1 & 6 & 5
\end{array}\right)
$$

We note that the two subsets of indices, $\{1,3,5\}$ and $\{2,4,6\}$, are each invariant under the permutation $\sigma$, and interchanged under $\tau$. To be precise,

$$
\sigma(1,3,5)=(5,3,1), \quad \sigma(2,4,6)=(4,2,6), \quad \tau(1,3,5)=(4,2,6), \quad \tau(2,4,6)=(3,1,5) .
$$

Some reflections resulting in particular symmetries are shown in Table 2.2, with notation interpreted in an obvious manner up to equation (2.3).

Concerning self-symmetry, we note the following property.

TABLE 2.2. Examples of reflections between two regular hexagons at standard position.

\begin{tabular}{lcc}
\hline Planar reflection & Axis: $y=x \tan \xi$ & Mapping relation \\
\hline With respect to $y=x$ & $\xi=\frac{\pi}{4}$ & $\left(x_{j}^{(\pi / 2-\varphi)}, y_{j}^{(\pi / 2-\varphi)}\right)=\left(y_{\sigma(j)}^{(\varphi)}, x_{\sigma(j)}^{(\varphi)}\right)$ \\
With respect to $y=-x$ & $\xi=\frac{3 \pi}{4}$ & $\left(x_{j}^{(3 \pi / 2-\varphi)}, y_{j}^{(3 \pi / 2-\varphi)}\right)=\left(-y_{\sigma(j)}^{(\varphi)},-x_{\sigma(j)}^{(\varphi)}\right)$ \\
With respect to $y=0$ & $\xi=0$ & $\left(x_{j}^{(-\varphi)}, y_{j}^{(-\varphi)}\right)=\left(x_{\sigma(j)}^{(\varphi)},-y_{\sigma(j)}^{(\varphi)}\right)$ \\
With respect to $x=0$ & $\xi=\frac{\pi}{2}$ & $\left(x_{j}^{(\pi-\varphi)}, y_{j}^{(\pi-\varphi)}\right)=\left(-x_{\sigma(j)}^{(\varphi)}, y_{\sigma(j)}^{(\varphi)}\right)$ \\
\hline
\end{tabular}


Property 2.3 (Dihedral reflections on a regular hexagon). Among planar reflections with respect to lines $y=x \tan \xi, \xi=\varphi+\pi / 3+k \pi / 6$, the choices $k=1,3,5$ yield on the same hexagon at standard position the three median reflections, and $k=0,2,4$ the three (long-) diagonal reflections.

We state our main result.

TheOREm 2.4 (Hex-seven-point cubature rule for a regular hexagon in arbitrary phase). The expression

$$
\frac{1}{|\Omega|} \iint_{\Omega} f(x, y) d x d y=\frac{31}{36} f\left(P_{0}\right)+\frac{5}{36} \frac{1}{6} \sum_{i=1}^{6} f\left(P_{i}\right)+\mathcal{O}\left(h^{4}\right)
$$

is valid for $f \in C^{6}$.

\section{Integrals in a hexagon}

To investigate the discretization of a definite integral in a hexagon with phase angle $\varphi$, we assume $P_{0}=(0,0)$ and consider the average central moment (integral)

$$
M_{m, n}^{\varphi}:=\frac{1}{|\Omega|} \iint_{\Omega} x^{m} y^{n} d x d y=\frac{\sqrt{3}}{6 h^{2}} \sum_{l=1}^{6} \iint_{\Omega_{l}} x^{m} y^{n} d x d y, \quad 0 \leqslant m, n \in \mathbb{Z} .
$$

Lemma 3.1. The moments $\left(M_{m, n}^{\varphi}\right)$ vanish in all phases, if $m+n$ is odd.

Proof. Just by the domain symmetry (Property 2.1),

$$
\iint_{\Omega_{l+3}} x^{m} y^{n} d x d y=(-1)^{m+n} \iint_{\Omega_{l}} x^{m} y^{n} d x d y, \quad l=1,2,3
$$

More numerical values and qualitative properties of the moments are derived in the sequel.

\subsection{Moments by secant integrals}

Lemma 3.2. Assume that both $m$ and $n$ are odd. The moments $M_{m, n}^{\varphi}$ in equation (3.1) vanish, either with a type I or II hexagon, or with a general phase angle and $m+n<6$.

Proof. With $\varphi=0$ or $-\pi / 6$, the first part of the assertion is implied by the domain symmetry. In the general case, a moment can be expressed (using polar coordinates and $\left\|P_{0} P_{\theta}\right\|=h \sec \theta$ in Figure 3.1) as

$$
\begin{aligned}
M_{m, n}^{\varphi} & =\frac{\sqrt{3}}{6 h^{2}} \sum_{l=1}^{6} \int_{-\pi / 6}^{\pi / 6} \int_{0}^{h \sec \theta} \cos ^{m}\left(\theta+\psi+\frac{l \pi}{3}\right) \sin ^{n}\left(\theta+\psi+\frac{l \pi}{3}\right) \rho^{m+n+1} d \rho d \theta \\
& =\frac{\sqrt{3} h^{m+n}}{6(m+n+2)} \int_{-\pi / 6}^{\pi / 6} \sec ^{m+n+2} \theta S_{m, n}^{\varphi}\left(\theta+\frac{\pi}{6}\right) d \theta,
\end{aligned}
$$

in which, with $\xi=\theta+\pi / 6$,

$$
S_{m, n}^{\varphi}(\xi):=\sum_{l=1}^{6} \cos ^{m}\left(\xi+\varphi+\frac{l \pi}{3}\right) \sin ^{n}\left(\xi+\varphi+\frac{l \pi}{3}\right) .
$$

The fact that $S_{1,1}^{\varphi}(\xi)=S_{3,1}^{\varphi}(\xi)=S_{1,3}^{\varphi}(\xi)=0$ for all $\varphi$ and $\xi$, as shown in the Appendix, completes the proof. 


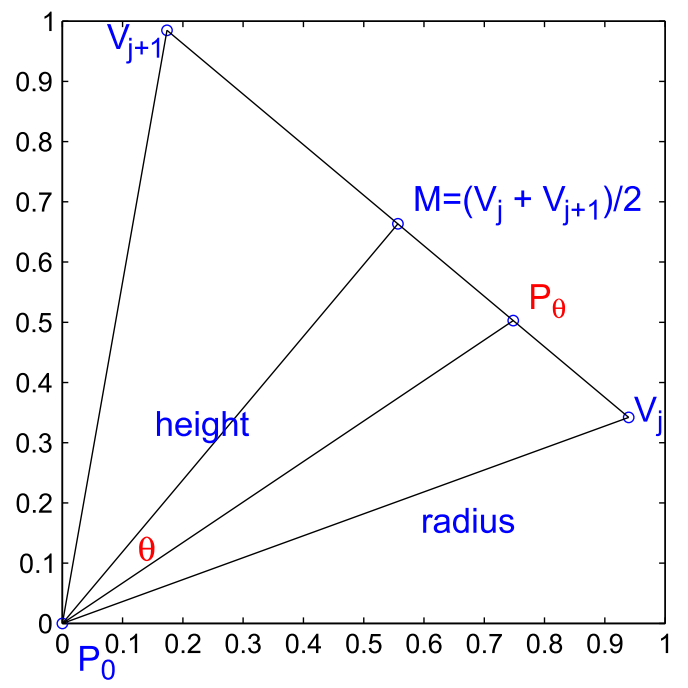

FIgURE 3.1. Local coordinates at component triangles of a regular hexagon.

We note that if $m+n$ is odd, then $S_{m, n}^{\varphi}=0$, as it should be and is easily seen.

Lemma 3.3. With fixed nonnegative integers $m$ and $n$, if $S_{m, n}^{\varphi}(\xi)$ is independent of $\varphi$ and $\xi$, then $S_{n, m}=S_{m, n}$ as a constant.

Proof. It is straightforward to see that

$$
\begin{aligned}
S_{m, n}^{\pi / 2-\varphi}(\xi) & =\sum_{l=1}^{6} \cos ^{m}\left(\xi+\frac{\pi}{2}-\varphi+\frac{l \pi}{3}\right) \sin ^{n}\left(\xi+\frac{\pi}{2}-\varphi+\frac{l \pi}{3}\right) \\
& =\sum_{l=1}^{6} \sin ^{m}\left(\varphi-\xi+\frac{(6-l) \pi}{3}\right) \cos ^{n}\left(\varphi-\xi+\frac{(6-l) \pi}{3}\right)=S_{n, m}^{\varphi}(-\xi)
\end{aligned}
$$

Hence the result.

More moments can be determined by using

$$
\begin{gathered}
\int \sec ^{k} \theta d \theta=\frac{k-2}{k-1} \int \sec ^{k-2} \theta d \theta+\frac{1}{k-1} \tan \theta \sec ^{k-2} \theta, \quad 1<k \in \mathbb{N}, \\
\int_{-\pi / 6}^{\pi / 6} \sec ^{k} \theta d \theta=\frac{2}{\sqrt{3}}, \frac{20}{9 \sqrt{3}}, \frac{112}{45 \sqrt{3}}, \quad \text { for } k=2,4,6, \text { respectively. }
\end{gathered}
$$

Equations (3.2)-(3.4) together give explicit (recursive) expressions for low-degree moments. These are collected in Table 3.1 .

\section{Hexagonal integral stencil}

To prove the main result (Theorem 2.4), we establish the relation

$$
\sum_{j=0}^{6} w_{j} f\left(P_{j}\right) \approx \frac{1}{|\Omega|} \iint_{\Omega} f(x, y) d x d y
$$


by obtaining the weights $\left\{w_{j}\right\}_{j=0}^{6}$ using low-degree shifted monomials in the form $f(x, y)=$ $\left(x-x_{0}\right)^{m}\left(y-y_{0}\right)^{n}$. Without loss of generality, we assume the reduction $x_{0}=y_{0}=0$ in equation (2.1), and observe on monomials $f=x^{m} y^{n}$ that $f_{P_{j+3}}=(-1)^{m+n} f_{P_{j}}, j=1,2,3$. For convenience, the interpolation conditions to determine the weights are collected in Table 4.1 with the right-hand-side (RHS) values taken from Table 3.1.

We give a brief discussion.

(1) The first integrand $(m=n=0, f=1)$ yields $\sum_{0}^{6} w_{j}=1$, or $w_{0}=-\sum_{1}^{6} w_{j}$. All other $((m, n) \neq(0,0))$ conditions involve only $\left\{w_{j}\right\}_{1}^{6}$ since $P_{0}=(0,0)$.

(2) The cases of linear monomials, $f \in\{x, y\}$, imply

$$
\left[\begin{array}{lll}
x_{1} & x_{3} & x_{5} \\
y_{1} & y_{3} & y_{5}
\end{array}\right] \cdot\left[\begin{array}{l}
w_{1}-w_{4} \\
w_{3}-w_{6} \\
w_{5}-w_{2}
\end{array}\right]=\left[\begin{array}{l}
0 \\
0
\end{array}\right]
$$

or, by using $P_{5}=-P_{1}-P_{3}$,

$$
\left[\begin{array}{ll}
x_{1} & x_{3} \\
y_{1} & y_{3}
\end{array}\right] \cdot\left[\begin{array}{l}
w_{1}-w_{4}-\left(w_{5}-w_{2}\right) \\
w_{3}-w_{6}-\left(w_{5}-w_{2}\right)
\end{array}\right]=\left[\begin{array}{l}
0 \\
0
\end{array}\right]
$$

That $\left\{P_{0}, P_{1}, P_{3}\right\}$ are not colinear yields a (unique) trivial solution such that

$$
w_{1}-w_{4}=w_{5}-w_{2}=w_{3}-w_{6}=\delta_{w} \text { is a constant }
$$

(3) In the four cubic cases, $f \in\left\{x^{3}, y^{3}, x^{2} y, x y^{2}\right\}$, we obtain

$$
M_{3} \cdot\left[\begin{array}{l}
w_{1}-w_{4} \\
w_{3}-w_{6} \\
w_{5}-w_{2}
\end{array}\right] \equiv\left[\begin{array}{ccc}
x_{1}^{3} & x_{3}^{3} & x_{5}^{3} \\
y_{1}^{3} & y_{3}^{3} & y_{5}^{3} \\
x_{1}^{2} y_{1} & x_{3}^{2} y_{3} & x_{5}^{2} y_{5} \\
x_{1} y_{1}^{2} & x_{3} y_{3}^{2} & x_{5} y_{5}^{2}
\end{array}\right] \cdot\left[\begin{array}{l}
w_{1}-w_{4} \\
w_{3}-w_{6} \\
w_{5}-w_{2}
\end{array}\right]=\left[\begin{array}{l}
0 \\
0 \\
0 \\
0
\end{array}\right]
$$

The elementary column operation on $M_{3}$, adding the first two columns to the third,

TABLE 3.1. Average central moments of a phase- $\varphi$ regular hexagon.

\begin{tabular}{ccccc}
\hline$m$ & $n$ & $S_{m, n}^{\varphi}$ & $M_{m, n}^{\varphi}$ & Remark \\
\hline Even & Odd & 0 & 0 & \\
Odd & Even & 0 & 0 & Type I, II, or $m+n<6$ \\
Odd & Odd & 0 & 0 & \\
0 & 2 & 3 & $\frac{5}{18} h^{2}\left(=\frac{5}{24} r^{2}\right)$ & \\
2 & 0 & 3 & $\frac{5}{18} h^{2}\left(=\frac{5}{24} r^{2}\right)$ & \\
0 & 4 & $\frac{9}{4}$ & $\frac{7}{45} h^{4}\left(=\frac{7}{60} r^{4}\right)$ & \\
4 & 0 & $\frac{9}{4}$ & $\frac{7}{45} h^{4}\left(=\frac{7}{60} r^{4}\right)$ & \\
2 & 2 & $\frac{3}{4}$ & $\frac{7}{135} h^{4}\left(=\frac{7}{180} r^{4}\right)$ & \\
\hline
\end{tabular}


yields (by Lemma A.1)

$$
\left[\begin{array}{ccc}
x_{1}^{3} & x_{3}^{3} & \frac{3 d^{3}}{4} \sin 3 \varphi \\
y_{1}^{3} & y_{3}^{3} & \frac{3 d^{3}}{4} \cos 3 \varphi \\
x_{1}^{2} y_{1} & x_{3}^{2} y_{3} & -\frac{3 d^{3}}{4} \cos 3 \varphi \\
x_{1} y_{1}^{2} & x_{3} y_{3}^{2} & -\frac{3 d^{3}}{4} \sin 3 \varphi
\end{array}\right] \cdot\left[\begin{array}{c}
w_{1}-w_{4}-\left(w_{5}-w_{2}\right) \\
w_{3}-w_{6}-\left(w_{5}-w_{2}\right) \\
w_{5}-w_{2}
\end{array}\right]=\left[\begin{array}{l}
0 \\
0 \\
0 \\
0
\end{array}\right]
$$

which, together with

$$
\left(w_{1}-w_{4}-\left(w_{5}-w_{2}\right), w_{3}-w_{6}-\left(w_{5}-w_{2}\right), w_{5}-w_{2}\right)=\left(0,0, \delta_{w}\right)
$$

TABLE 4.1. Interpolation conditions (equation (4.1)) on monomials for seven-point cubature on a regular hexagon. Stated expressions depend only on $x_{0}=y_{0}=0$ and the domain symmetry. In

\begin{tabular}{|c|c|c|c|}
\hline$m, n$ & $f(x, y)$ & $\mathrm{LHS}=\sum_{j=0}^{6} w_{j} f_{P_{j}}$ & $\mathrm{RHS}=M_{m, n}^{\varphi}=\frac{1}{|\Omega|} \iint_{\Omega} f d x d y$ \\
\hline 0,0 & 1 & $\sum_{0}^{6} w_{j}$ & 1 \\
\hline 1,0 & $x$ & $\sum_{j=1,3,5}\left(w_{j}-w_{j+3}\right) x_{j}$ & 0 \\
\hline 0,1 & $y$ & $\sum_{j=1,3,5}\left(w_{j}-w_{j+3}\right) y_{j}$ & 0 \\
\hline 2,0 & $x^{2}$ & $\sum_{j=1,3,5}\left(w_{j}+w_{j+3}\right) x_{j}^{2}$ & $\frac{5 r^{2}}{24}\left(=\frac{5 d^{2}}{72}\right)$ \\
\hline 0,2 & $y^{2}$ & $\sum_{j=1,3,5}\left(w_{j}+w_{j+3}\right) y_{j}^{2}$ & $\frac{5 r^{2}}{24}\left(=\frac{5 d^{2}}{72}\right)$ \\
\hline 1,1 & $x y$ & $\sum_{j=1,3,5}\left(w_{j}+w_{j+3}\right) x_{j} y_{j}$ & 0 \\
\hline 3,0 & $x^{3}$ & $\sum_{j=1,3,5}\left(w_{j}-w_{j+3}\right) x_{j}^{3}$ & 0 \\
\hline 0,3 & $y^{3}$ & $\sum_{j=1,3,5}\left(w_{j}-w_{j+3}\right) y_{j}^{3}$ & 0 \\
\hline 2,1 & $x^{2} y$ & $\sum_{j=1,3,5}\left(w_{j}-w_{j+3}\right) x_{j}^{2} y_{j}$ & 0 \\
\hline 1,2 & $x y^{2}$ & $\sum_{j=1,3,5}\left(w_{j}-w_{j+3}\right) x_{j} y_{j}^{2}$ & 0 \\
\hline$m+n$ odd & $x^{m} y^{n}$ & $\sum_{j=1,3,5}\left(w_{j}-w_{j+3}\right) x_{j}^{m} y_{j}^{n}$ & 0 \\
\hline
\end{tabular}
particular, $P_{j+3}=-P_{j}, j=1,2,3$. 
(by equation (4.2)), implies $\delta_{w}=0$. Otherwise we would obtain $\sin 3 \varphi=0$ but also $\cos 3 \varphi=0$, a contradiction. The stencil is therefore symmetric such that

$$
w_{j}=w_{j+3}, \quad j=1,2,3 .
$$

(4) The cases $u \in\left\{x^{2}, y^{2}, x y\right\}$ yield

$$
M_{\mathrm{sym}} \cdot w^{s y m} \equiv\left[\begin{array}{ccc}
x_{1}^{2} & x_{3}^{2} & x_{5}^{2} \\
y_{1}^{2} & y_{3}^{2} & y_{5}^{2} \\
2 x_{1} y_{1} & 2 x_{3} y_{3} & 2 x_{5} y_{5}
\end{array}\right] \cdot\left[\begin{array}{l}
w_{1}+w_{4} \\
w_{3}+w_{6} \\
w_{5}+w_{2}
\end{array}\right]=\frac{5 d^{2}}{72}\left[\begin{array}{l}
1 \\
1 \\
0
\end{array}\right] .
$$

It can be shown that

$$
M_{\mathrm{sym}}^{-1}=\frac{2}{3 d^{2}}\left[\begin{array}{ccc}
\frac{1}{2}-\cos 2 \varphi & \frac{1}{2}+\cos 2 \varphi & -\sin 2 \varphi \\
\frac{1}{2}-\cos \left(2 \varphi-\frac{2 \pi}{3}\right) & \frac{1}{2}+\cos \left(2 \varphi-\frac{2 \pi}{3}\right) & -\sin \left(2 \varphi-\frac{2 \pi}{3}\right) \\
\frac{1}{2}-\cos \left(2 \varphi+\frac{2 \pi}{3}\right) & \frac{1}{2}+\cos \left(2 \varphi+\frac{2 \pi}{3}\right) & -\sin \left(2 \varphi+\frac{2 \pi}{3}\right)
\end{array}\right] .
$$

Therefore

$$
\left[\begin{array}{l}
w_{1}+w_{4} \\
w_{3}+w_{6} \\
w_{5}+w_{2}
\end{array}\right]=M_{\mathrm{sym}}^{-1} \cdot \frac{5 d^{2}}{72}\left[\begin{array}{l}
1 \\
1 \\
0
\end{array}\right]=\frac{10}{216}\left[\begin{array}{l}
1 \\
1 \\
1
\end{array}\right]
$$

Finally,

$$
w_{1}=w_{4}=w_{3}=w_{6}=w_{5}=w_{2}=\frac{5}{216}, \quad w_{0}=\frac{31}{36} .
$$

The cubature rule (equations (4.1) and (4.3)) so determined is exact on all odd-degree monomials by the symmetry in both the integral stencil and the domain. The stencil is of at least fourth order by construction. That it is indeed of fourth order is justified as follows: (i) the weights are exact on degree-five monomials (Table 3.1); (ii) on the three degree-four even monomials $\left\{x^{4}, y^{4}, x^{2} y^{2}\right\}$, deviations in the discrete approximation (equations (4.1) and (4.3)) from the theoretic values (Table 3.1) are, respectively,

$$
\frac{7}{45} h^{4}-\frac{5}{216} S_{4,0} d^{4}\left(=\frac{-61}{90} h^{4}\right), \quad \frac{-61}{90} h^{4}, \quad \frac{7}{135} h^{4}-\frac{5}{216} S_{2,2} d^{4} \quad\left(=\frac{-61}{270} h^{4}\right) .
$$

Thus completes the proof of Theorem 2.4.

\section{Conclusions}

Irregular domains approximated by nets of regular hexagons are found advantageous in the study of biological wave phenomena in recent research. In this work, central moments on regular hexagons are resolved and a fourth-order seven-point cubature is developed for regular hexagons, using interior and neighbor center nodes. The hexagonal seven-point cubature is basic to further applications of integrals on hexagons or approximated domains.

\section{Appendix. Some low-degree barycentric identities}

The technical details supporting $\S \S 3.1$ and 4 are presented here. Let $\psi=\varphi+\pi / 6, d=1.0$, and

$$
P_{0}=(0,0), \quad P_{j}=\left(x_{j}, y_{j}\right)=\left(\cos \xi_{j}, \sin \xi_{j}\right), \quad \xi_{j}=\psi+\frac{j \pi}{3}, \quad j=1,3,5 .
$$


Lemma A.1. For a regular hexagon centered at the origin with an arbitrary phase angle $\varphi$, the following identities hold.

$$
\begin{gathered}
x_{1}+x_{3}+x_{5}=y_{1}+y_{3}+y_{5}=0 . \\
x_{1}^{2}+x_{3}^{2}+x_{5}^{2}=y_{1}^{2}+y_{3}^{2}+y_{5}^{2}=\frac{3}{2} . \\
x_{1} y_{1}+x_{3} y_{3}+x_{5} y_{5}=0 . \\
x_{1}^{3}+x_{3}^{3}+x_{5}^{3}=-x_{1} y_{1}^{2}-x_{3} y_{3}^{2}-x_{5} y_{5}^{2}=-\frac{3}{4} \cos 3 \psi=\frac{3}{4} \sin 3 \varphi . \\
y_{1}^{3}+y_{3}^{3}+y_{5}^{3}=-x_{1}^{2} y_{1}-x_{3}^{2} y_{3}-x_{5}^{2} y_{5}=\frac{3}{4} \sin 3 \psi=\frac{3}{4} \cos 3 \varphi . \\
x_{1}^{4}+x_{3}^{4}+x_{5}^{4}=y_{1}^{4}+y_{3}^{4}+y_{5}^{4}=\frac{9}{8} . \\
x_{1}^{2} y_{1}^{2}+x_{3}^{2} y_{3}^{2}+x_{5}^{2} y_{5}^{2}=\frac{3}{8} . \\
x_{1}^{3} y_{1}+x_{3}^{3} y_{3}+x_{5}^{3} y_{5}=x_{1} y_{1}^{3}+x_{3} y_{3}^{3}+x_{5} y_{5}^{3}=0 .
\end{gathered}
$$

Proof. The linear case follows by the geometric argument that

$$
P_{1}+P_{3}+P_{5}=3 P_{0}=(0,0) .
$$

Various real arguments exist for the cubic identities; for a simple complex one, just note

$$
\begin{aligned}
z & \equiv\left(x_{1}+i y_{1}\right)^{3}+\left(x_{3}+i y_{3}\right)^{3}+\left(x_{5}+i y_{5}\right)^{3} \\
& =e^{i 3 \xi_{1}}+e^{i 3 \xi_{3}}+e^{i 3 \xi_{5}}=3 e^{i 3 \psi} e^{\pi i}=-3(\cos 3 \psi+i \sin 3 \psi),
\end{aligned}
$$

and

$$
\begin{aligned}
& -3 \cos 3 \psi=\Re z=x_{1}^{3}+x_{3}^{3}+x_{5}^{3}-3\left(x_{1} y_{1}^{2}+x_{3} y_{3}^{2}+x_{5} y_{5}^{2}\right), \\
& -3 \sin 3 \psi=\Im z=-y_{1}^{3}-y_{3}^{3}-y_{5}^{3}+3\left(x_{1}^{2} y_{1}+x_{3}^{2} y_{3}+x_{5}^{2} y_{5}\right),
\end{aligned}
$$

in addition to

$$
\begin{aligned}
\left(x_{1}^{3}+x_{3}^{3}+x_{5}^{3}\right)+\left(x_{1} y_{1}^{2}+x_{3} y_{3}^{2}+x_{5} y_{5}^{2}\right) & =x_{1}\left(x_{1}^{2}+y_{1}^{2}\right)+x_{3}\left(x_{3}^{2}+y_{3}^{2}\right)+x_{5}\left(x_{5}^{2}+y_{5}^{2}\right)=0, \\
\left(y_{1}^{3}+y_{3}^{3}+y_{5}^{3}\right)+\left(y_{1} x_{1}^{2}+y_{3} x_{3}^{2}+y_{5} x_{5}^{2}\right) & =y_{1}\left(x_{1}^{2}+y_{1}^{2}\right)+y_{3}\left(x_{3}^{2}+y_{3}^{2}\right)+y_{5}\left(x_{5}^{2}+y_{5}^{2}\right)=0 .
\end{aligned}
$$

We proceed differently for the other cases, which all depend on the linear result.

With $\alpha=\xi+\varphi$ in equation (3.3), we note for equations (A.2) and (A.3),

$$
\begin{gathered}
S_{2,0}^{\varphi}(\xi)=\sum_{j=1}^{6} \begin{array}{c}
\cos ^{2} \\
\sin ^{2}
\end{array}\left(\alpha+\frac{j \pi}{3}\right)=\frac{1}{2} \sum_{j=1}^{6}\left(1 \pm \cos \left(2 \alpha+\frac{2 j \pi}{3}\right)\right)=3, \\
S_{0,2}^{\varphi}(\xi)(\xi)=\sum_{j=1}^{6} \cos \left(\alpha+\frac{j \pi}{3}\right) \sin \left(\alpha+\frac{j \pi}{3}\right)=\frac{1}{2} \sum_{j=1}^{6} \sin \left(2 \alpha+\frac{2 j \pi}{3}\right)=0 ;
\end{gathered}
$$

for equation (A.6),

$$
\begin{aligned}
S_{4,0}^{\varphi}(\xi) & =\sum_{j=1}^{6} \underset{\sin ^{4}}{\cos ^{4}}\left(\alpha+\frac{j \pi}{3}\right)=\sum_{j=1}^{6}\left(\frac{1 \pm \cos (2 \alpha+(2 j \pi) / 3)}{2}\right)^{2} \\
& =\frac{1}{4} \sum_{j=1}^{6}\left(1 \pm 2 \cos \left(2 \alpha+\frac{2 j \pi}{3}\right)+\frac{1+\cos (4 \alpha+(4 j \pi) / 3)}{2}\right)=\frac{6}{4}+\frac{6}{8}=\frac{9}{4}
\end{aligned}
$$


and for equation (A.8),

$$
\begin{aligned}
S_{3,1}^{\varphi}(\xi) S_{1,3}^{\varphi}(\xi) & =\sum_{j=1}^{6} \cos ^{3}\left(\alpha+\frac{j \pi}{3}\right) \sin \left(\alpha+\frac{j \pi}{3}\right) \\
& =\frac{1}{4} \sum_{j=1}^{6}\left(1 \pm \cos \left(2 \alpha+\frac{2 j \pi}{3}\right)\right) \sin \left(2 \alpha+\frac{2 j \pi}{3}\right) \\
& =\frac{1}{4} \sum_{j=1}^{6} \sin \left(2 \alpha+\frac{2 j \pi}{3}\right) \pm \frac{1}{8} \sum_{j=1}^{6} \sin \left(4 \alpha+\frac{4 j \pi}{3}\right)=0 .
\end{aligned}
$$

Finally, the fact that

$$
6=\sum_{j=1}^{6}\left(\cos ^{2}\left(\alpha+\frac{j \pi}{3}\right)+\sin ^{2}\left(\alpha+\frac{j \pi}{3}\right)\right)^{2}=S_{4,0}^{\varphi}(\xi)+2 S_{2,2}^{\varphi}(\xi)+S_{0,4}^{\varphi}(\xi),
$$

yields $S_{2,2}^{\varphi}(\xi)=\frac{3}{4}$ for equation (A.7). This concludes the proof.

REMARK A.2.

(1) Actually, $S_{0,2}=S_{2,0}, S_{0,4}=S_{4,0}, S_{1,3}=S_{3,1}$, by Lemma 3.3.

(2) Except for equations (A.3) and (A.7), one half of the barycentric identities are implied by the other half, using the symmetry argument (the first example in Table 2.2) that

$$
\left\{\left(x_{1}, x_{3}, x_{5}\right),\left(y_{1}, y_{3}, y_{5}\right)\right\} \text { are congruent to }\left\{\left(y_{5}, y_{3}, y_{1}\right),\left(x_{5}, x_{3}, x_{1}\right)\right\} \text {. }
$$

Note also for equations (A.4) and (A.5) that

$$
\cos \left(3\left(\frac{\pi}{2}-\psi\right)\right)=-\sin 3 \psi \quad \text { and } \quad \sin \left(3\left(\frac{\pi}{2}-\psi\right)\right)=-\cos 3 \psi
$$

Alternatively, the equivalence within the pair of cubic identities comes from the second example in Table 2.2.

(3) All the claimed identities are valid with the indices $\{1,3,5\}$ replaced by $\{2,4,6\}$, except for negating the constants on the RHSs of the cubic cases. This corresponds to replacing $\varphi$ by $\varphi-\pi / 3$ (rotational symmetry, not explicitly mentioned).

Acknowledgements. The authors are grateful to Tunghai University and Providence University for the continued laboratory support.

\section{References}

1. J. Berntsen and T. O. Espelid, 'Algorithm 706, DCUTRI: an algorithm for adaptive cubature over a collection of triangles', ACM TOMS 18 (1992) no. 3, 329-342.

2. R. Cools, 'Monomial cubature rules since "Stroud": a compilation-part 2', J. Comput. Appl. Math. 112 (1999) 21-27.

3. R. Cools, 'An encyclopaedia of cubature formulas', J. Complexity 19 (2003) 445-453.

4. R. Cools, D. Laurie and L. Pluym, 'Algorithm 764: Cubpack++: A C++ package for automatic twodimensional cubature', ACM TOMS 23 (1997) no. 1, 1-15.

5. R. Cools and P. Rabinowitz, 'Monomial cubature rules since "Stroud": a compilation', J. Comput. Appl. Math. 48 (1993) 309-326.

6. H. J. Ritsema van Eck, J. A. Kors and G. van Herpen, 'The U wave in the electrocardiogram: a solution for a 100-year-old riddle', Cardiovasc Res. 67 (2005) no. 2, 256-262.

7. T. HAhn, 'CUBA - a library for multidimensional numerical integration', Comput. Phys. Commun. 168 (2005) 78-95. 
8. D. Lee, H. C. Tien, C. P. Luo and H.-N. Luk, 'Hexagonal grid methods with applications to partial differential equations', Int. J. Comput. Math. 91 (2014) 1986-2009.

9. J. N. Lyness and R. Cools, 'A survey of numerical cubature over triangles', Preprint MCS-P410-0194, Argonne National Laboratory, Argonne, IL, 1994.

\section{Daniel Lee}

Department of Applied Mathematics

Tunghai University

Taichung 40704

Taiwan

danlee@thu.edu.tw

\author{
Hui-Chun Tien \\ Department of Financial and \\ Computational Mathematics \\ Providence University \\ Taichung 40704 \\ Taiwan \\ hctien@pu.edu.tw
}

